\title{
Product Identification System Using Block chain
}

\section{Sushil Kumar ${ }^{1}$, Vikas Gupta ${ }^{1}$, Yash Pachori ${ }^{1}$, Rishabh Sharma ${ }^{1}$, Anshika Agarwal ${ }^{1}$}

${ }^{1}$ Department of Computer Science and Engineering, Krishna Engineering College, Ghaziabad, India

\begin{abstract}
Article Info

Volume 7, Issue 3

Page Number: 258-261

Publication Issue :

May-June-2021

\section{Article History}

Accepted : 15 May 2021

Published : 22 May 2021

In recent years, block chain has received increasing attention and various applications have emerged from this technology. A renowned Blockchain application is that the cryptocurrency Bitcoin, that has not only been effectively solving the double-spending problem but also it can confirm the legitimacy of transactional records without counting on a centralized system to try and do so. Therefore, any application using Blockchain technology because the base architecture ensures that the contents of its data are tamper-proof. This paper uses the decentralized Blockchain technology approach to confirm that buyers don't fully depend on the merchants to work out if products are genuine. We describe a decentralized Blockchain system with products anti-counterfeiting; therein way manufacturers can use this method to supply genuine products without having to manage direct-operated stores, which may significantly reduce the value of product quality assurance.
\end{abstract}

Keywords : Blockchain, Counterfeit

\section{INTRODUCTION}

There are many fake products in environment and to confirm that the aptitude of products system is required. The exchange counterfeit products and affecting the sales and profits of companies suffering from this phenomenon to confirm the identification and traceability of real products in supply-chain, this paper is propose a completely functional Blockchain system to stop product counterfeiting small and medium-sized enterprises (SMEs) often have financial burdens, which can't be compared with large companies with strong financial resources within the brand management sector, SMEs will inevitably have to reduce costs and can be presumably unable to implement traditional methods of preventing counterfeited goods. In this paper, we've got implement a Blockchain architecture provided to record product ownership on the Blockchain. By using Block chain's intractability and transparency properties, and therefore the assurance that every record cannot be forged on the Block chain consumers don't must fully depend upon trusted third parties to soundly know the source of the purchased product. SMEs can implement the merchandise identification system during this paper, and that they will only must pay a comparatively low-cost for the operation fees to realize secure and genuine product authentication.

\section{RELATED WORK}


Here we have Blockchain based system are gradually being developed. We will focus on a product identification system; the recording data process on the supply chain is similar to our system's recorded information. The preceding research in identification of products based on Blockchain is discussed as follows. In the authors provide the design principles for products identification on Blockchain. They indicated that counterfeit products are important issues that brands with supply chain networks always need to acknowledge. By applying Blockchain in the product supply chain data records, they can strictly monitor the flow of products. We claims that the Blockchain characteristics, the information recorded on the Blockchain can be completely secure and trusted.

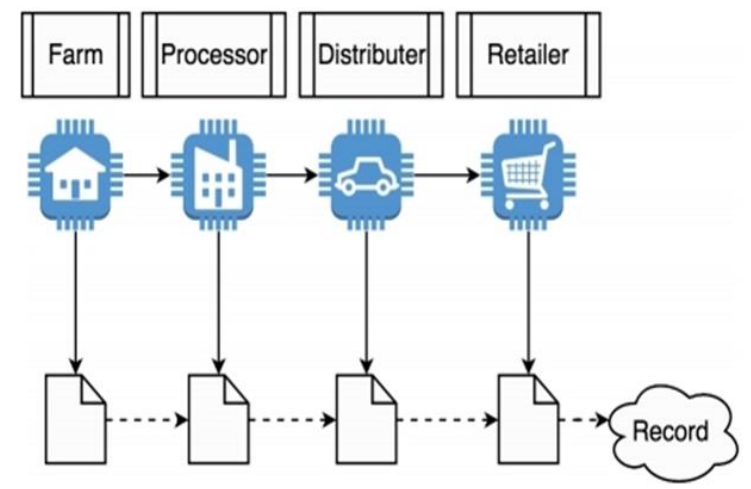

Fig. Product Supply data record on Block chain

\section{OPERATION FLOW}

In our design, the manufacturer is responsible for pushing seller information to the contract, including the number of products the seller can sell and the seller address. After the seller obtains the manufacturer's authorization, he can obtain a certain amount of recording rights for the products that he can sell on the contract. When the consumer purchases the product, the seller stores the consumer's address via the system to the contract to complete the transaction. Consumers can use the system to directly search for whether the seller is in the contract and whether there are unsold products available for trading. After purchasing, the consumer will provide the manufacturer with the information that the product wants to mail and encrypt it with the consumer's own private key. The manufacturer receives the encrypted data and will use the consumer's public key to restore it. If the information is consistent with consumer information, the manufacturer will send the product to the consumer and complete the shopping process.

\section{IMPLEMENTATION}

\section{Data Provider}

- Load Records

- Key Generation

- Encrypt Records(Attribute Based Encryption scheme using AES 128 )

- ${ }^{*}$ Blockchain Creation (Using bcprov-jdk16-140 jarfile )

- Upload

- Decrypt and Download Records

\section{Cloud Service Provider}

- View Records

- Grant or Revoke Permission

3. Language: Java.

4. Front End: Net beans 8.0.2 (JFrame).

5. Back End: $M y S Q L$.

6. Server: Wampserver.

\section{SYSTEMDESIGN}

We propose a non-productive and comprehensive counterfeit anti-fraud program, supported the Block chain. In our scheme, manufacturer scanning is employed by this program to store relevant information on the sale of products within the Block chain accessible to anyone the entire amount of sales which will be sold by these Leland and also the number of products currently left by this transparent 
pants. The user can use the functions provided by our system to perform verification on behalf of merchants, and this verification can't be performed.

\section{V.I.BASIC MODEL}

Our blockchain-based anti-fraud program consists of three parts, the Role of the Producer, the Retail Role, and also the client Role, we discuss the following:

1) Manufacturer's Role: On the seller's part, the services provided include a current vendor's address on contracts, add the quantity of products that retailers can sell, and return information to vendors to work out the foremost recent sales status. On the patron public key certificate it's possible to inquire about the merchandise these vendors are marketing to the patron, and verify if the merchandise has been changed or to verify that the present status of the merchandise has been verified by the client public key certificate.

2) Merchant Role: On behalf of the customer, these merchants may use program functions to encrypt the verification details of a non-public key, and thus the client may use the merchant public key to verify that this merchant is what he calls a stobe. After the acquisition and sale, these sellers specify the buyer's address within the agreement for the manufacturer to receive the knowledge. These retailers can access information about his products, like sales lists, and thus the quantity of his remaining stock.

3) Buyer's Role: during this a component of the vendor, the customer can verify that the vendor encompasses a sales relationship with the manufacturer and also to verify that the seller's stock has not been sold. On the a component of the manufacturer, consumer scans prove that their ownership is in step with their address and within the case of a well-maintained contract address, consumer scans receive records of every purchase and products status in their product.

\section{V.II. SYSTEM OPERATION}

1.) Login Process: Firstly, the user has to choose which account to log in. The user's accounts are connected to the Blockchain system, and the particular user can choose the account to log in their account by using Private key.

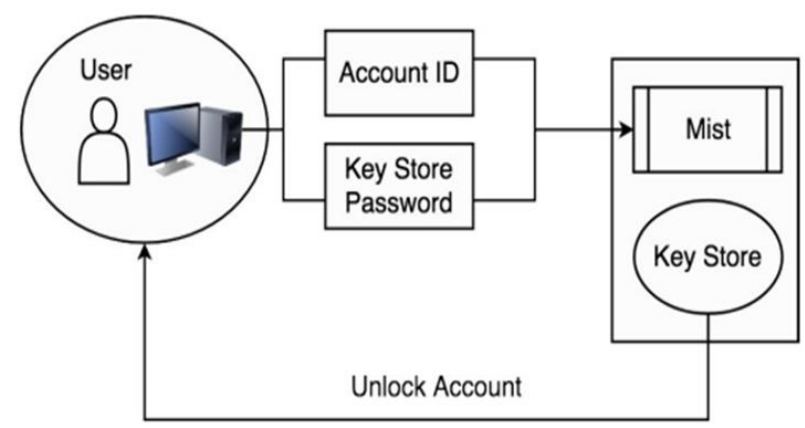

Fig. Log in process of our System

2.) Public Information of Contract: The information about the sellers is completely public. Our system provides smart contract data storage functions, which can provides seller list, consumer list, and remaining number of products Details of each seller.

3.) Adding New Seller and Product Number: The manufacturers can control the seller's details, and it also adding new seller and adding the number of genuine products that can be sold by the specific sellers.

4.) Recording the consumers on smart contract: The transaction between seller and consumer is established, the seller will add the consumers address in the smart contract.

5.) Identity Verification: The Identity Verification is most important feature in our system. Manufacturers verify the seller and consumers address who have added in smart contract. Our system allows users to use their private key to check their data and also provides the user's with a function on which they can verify one another. 
After the consumer acquires the essential information to verify the identity of the seller, the consumer will then call our system verification function to verify whether the seller identity is correct. After being added into contract product owner field by seller, the consumer can send the shipment address information which was encrypted by the consumer's private key and the hash value of the encrypted information to be verified by the manufacturer. The customer has to provide the seller address, the receiver name of the product, the mail address of the receivers, and the phone number of the receivers. The system will sign these data with the consumer's private key, and provide these data to the manufacturer.

\section{VI.CONCLUSION}

This Blockchain system that proposes a fully functional product genuineness system By paying a very low transaction fee, user's of our system no longer need to be concerned about the possibility of received a genuine and good quality products.

Manufacturers can use the system to store information of products will sale and buying in Blockchain which is accessible to everyone. This system provides Identity Verification by using transparency.

\section{REFERENCES}

[1]. J. Leng, P. Jiang, K. Xu, Q. Liu, J. L. Zhao, Y. Bian, and R. Shi, "Makerchain: A blockchain with chemical signature for self-organizing process in social manufacturing," J. Cleaner Prod., vol. 234, pp. 767-778, Oct. 2019.

[2]. N. Alzahrani and N. Bulusu, "Block-supply chain: A new anticounterfeiting supply chain using NFC and blockchain," in Proc. 1st Workshop Cryptocurrencies Blockchains Distrib. Syst. (CryBlock), 2018, pp. 30-35.
[3]. M. Rosenfeld (2012). [Online] Available: https://bitcoil.co.il/BitcoinX.

[4]. (2018). Litecoin. [Online]. Available: https://itecoin.info/index.php/Main Page

\section{Cite this Article}

Sushil Kumar, Vikas Gupta, Yash Pachori, Rishabh Sharma, Anshika Agarwal, "Product Identification System Using Block chain", International Journal of Scientific Research in Computer Science, Engineering and Information Technology (IJSRCSEIT), ISSN : 2456-3307, Volume 7 Issue 3, pp. 258-261, May-June 2021. Available at

doi : https://doi.org/10.32628/CSEIT217343

Journal URL : https://ijsrcseit.com/CSEIT217343 